\title{
Persisting unilateral lung hypoperfusion after resection of a subcarinal bronchogenic cyst
}

\author{
M. Barker*, G. Kusenbach*, E. Mühler**, E. Kleinhans+, G. Alzen++
}

Persisting unilateral lung hypoperfusion after resection of a subcarinal bronchogenic cyst. M. Barker, G. Kusenbach, E. Mühler, E. Kleinhans, G. Alzen. @ERS Journals Ltd 1996. ABSTRACT: As a rare malformation with a wide variety of clinical modes of presentation, the bronchogenic cyst remains a diagnostic challenge. We report a case with a subcarinal bronchogenic cyst and stenosis of the left main bronchus presenting as "unilateral hyperlucent lung".

Hypoplasia of the ipsilateral pulmonary vascular system persisted after surgical restitution of ventilation and is most likely due to an associated vascular malformation. Alternatively, anatomical fixation of pulmonary reflex vasoconstriction can be discussed.

Eur Respir J., 1996, 9, 844-845.

\author{
*Kinderklinik, **Klinik für Kinderkar- \\ diologie, +Klinik für Nuklearmedizin and \\ ${ }^{++}$Klinik für Radiologische Diagnostik, \\ Rheinisch-Westfälische Technische Hoch- \\ schule Aachen, Germany. \\ Correspondence: M. Barker, Kinderklinik, \\ Klinikum der RWTH Aachen, Pauwelsstraße \\ 30, D-52057 Aachen, Germany \\ Keywords: Bronchogenic cyst \\ pulmonary vascular hypoplasia \\ Received: August 11995 \\ Accepted for publication October 91995
}

Bronchogenic cysts are rare congenital foregut malformations arising from anomalous budding of the developing tracheobronchial tree. Depending on their location and size, the cysts can either remain asymptomatic or cause clinical complaints by displacing large airways, oesophagus and major blood vessels. The rate of inflammatory airway disease may be increased. Due to the risk of infection, haemorrhage, rupture or malignancy, the resection of a bronchogenic cyst soon after diagnosis is generally recommended, even in asymptomatic patients [1-3]. It is unclear whether the reversibility of functional impairment depends on the time of intervention.

We present the case of a boy, now 14 yrs of age, with the infrequent association of a mediastinal bronchogenic cyst and unilateral hypoplasia of the pulmonary vascular system.

\section{Case report}

A young boy suffered from recurrent airway infections since his first year of life. Neither dyspneoa, stridor nor chronic cough were noted, and his medical and family history as well as physical examination were unremarkable. A chest radiographic image obtained at the age of 5 yrs showed asymmetry of pulmonary vasculature with a hyperlucent left lung (fig. 1). Perfusion scan revealed a homogenous unilateral hypoperfusion with a left-toright ratio of $25: 75 \%$. By cardiac catheterization, a generalized hypoplasia of the left pulmonary blood vessels was found (fig. 2). Circumscript stenosis, pulmonary hypertension and intracardiac anomaly were excluded. The working diagnosis of Swyer-James syndrome was established.

Due to a decreasing rate of airway infections and absence of other symptoms, further diagnostic evaluation was delayed until the patient developed exercise dyspnoea at the age of 10 yrs. Repeated lung scanning confirmed hypoperfusion of the left lung (ratio 28:72\%), whilst revealing marked ipsilateral hypoventilation (10:90\%). On flexible fibreoptic bronchoscopy, a subtotal stenosis of the proximal left main bronchus was noted. Computed tomography (CT) (fig. 3.) and magnetic resonance imaging (MRI) scans of the chest demonstrated a $3 \times 4 \mathrm{~cm}$ cystic mass located directly below the tracheal bifurcation and compressing the left main bronchus. From a right lateral thoracotomy, the tumour was completely excised and classified as a bronchogenic cyst by pathological examination. The intra- and postoperative course was uncomplicated.

Two and a half years after surgery, follow-up studies by endoscopy, ventilation/perfusion scan and right heart catheterization were conducted because of subjective exercise intolerance. The central airways were fully patent, ventilation ratio had reached a normal value of $44: 56 \%$.

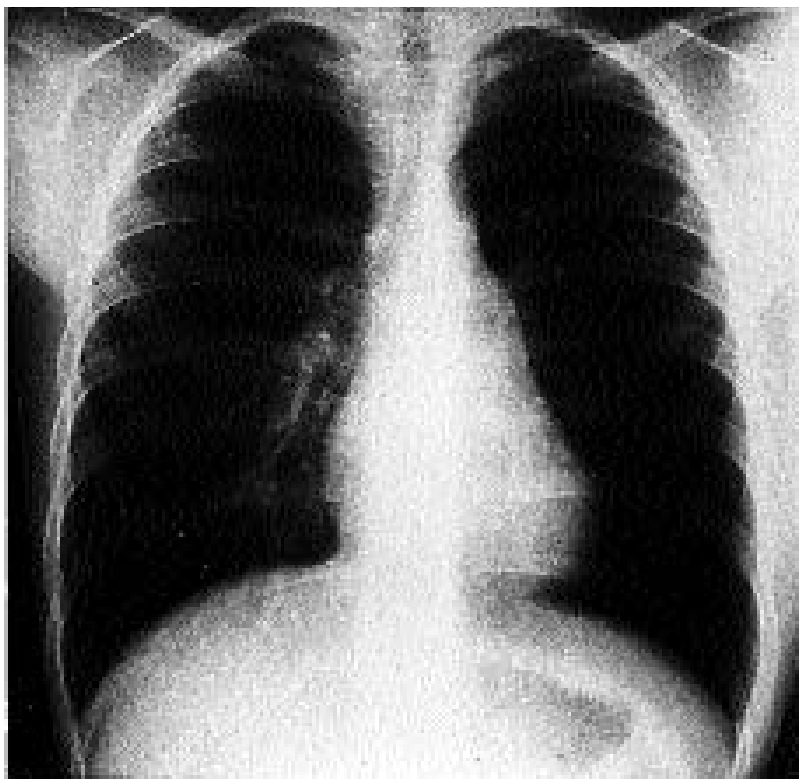

Fig. 1. - Chest radiograph, hyperlucent left lung. 


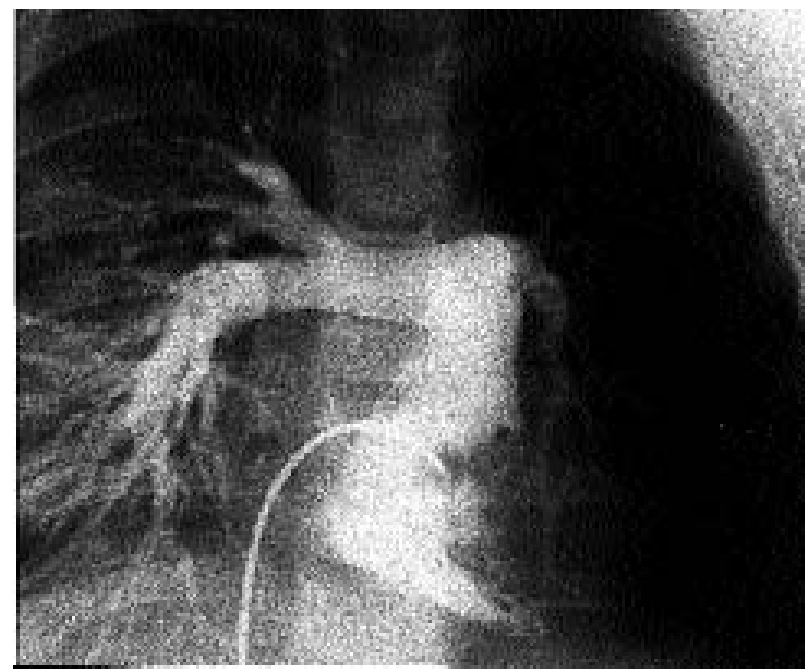

Fig. 2. - Pulmonary catheter angiography, hypoplasia of the left pulmonary vascular system.

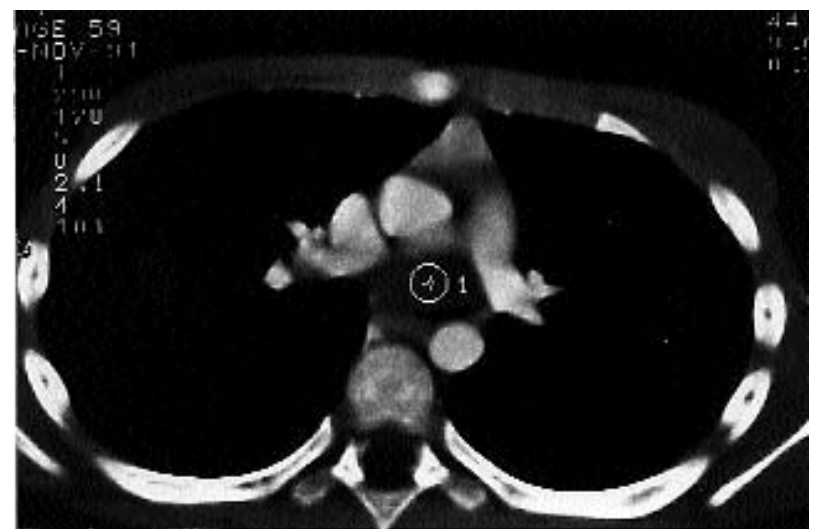

Fig. 3. - Computed tomography (CT) scan of the chest, subcarinal bronchogenic cyst marked (+), density 50 Houndsfield units.

Persistent findings were the unilateral hyperlucent lung on plain chest film, hypoperfusion of the left lung (ratio $32: 68 \%$ ) and hypoplasia of the left pulmonary vasculature as shown by angiography. Pulmonary arterial pressure was normal even after pharmacological provocation with orciprenaline. The patient's complaints could not be reproduced in a clinical exercise test and have not reappeared in the meantime.

\section{Discussion}

The case presented is definitely not unique for the late diagnosis of a central bronchogenic cyst negative on conventional chest radiographic image and with relatively few symptoms. The postoperatively persisting unilateral pulmonary hypoperfusion, however, is unusual and gives rise to the following considerations concerning aetiopathogenesis:

1. According to common knowledge of lung embryology and its disorders [4], it is feasible to assume that a cyst of early and central origin may mechanically obstruct blood flow to the left lung bud and, thereby, induce vascular hypoplasia. However, the absence of a circumscript stenosis of the pulmonary artery and normal development of all other lung structures render this hypothesis very unlikely. 2. A growing central cyst could compress the adjacent pulmonary artery and cause unilateral hypoperfusion. Such a case with a subcarinal bronchogenic cyst, pulmonary artery stenosis and unilateral perfusion defect has been described by BeRKOvITz et al. [5]. In their patient, however, ventilation of the affected lung was almost unimpaired and perfusion was completely restituted soon after resection of the cyst. Therefore, it seems improbable that compression of a central artery should lead to irreversible vascular hypoplasia in the dependent pulmonary blood vessels.

3. Another hypothesis for discussion is the anatomical fixation of pulmonary vasoconstriction mediated by the alveolocapillary reflex during years of severe unilateral hypoventilation. To our knowledge, such a mechanism has not been reported in other patients with unilateral bronchial obstruction or in an experimental setting, and so far our patient has not developed pulmonary hypertension. 4. Last but not least, a primary vascular malformation must be considered without direct causative connection to the bronchogenic cyst but possibly with a common teratogenetic factor. This aetiological concept was also mentioned by SELKE et al. [6], who, to our knowledge, published the only case with a similar combination of bronchogenic cyst and unilateral pulmonary vascular hypoplasia. The corresponding angiographic picture is nearly identical to that of our patient, but ventilation abnormalities were not reported.

Although the latter hypothesis appears most likely, the possibility of a direct relationship between the cyst and the vascular disorder cannot be completely ruled out in the case presented here. Suspicion remains as to whether early diagnosis and treatment might have prevented the postoperative persistence of pulmonary hypoperfusion.

In conclusion, the presented case of a bronchogenic cyst is characterized by the simultaneous findings of unilateral pulmonary hypoperfusion and hypoventilation. The persistence of hypoperfusion after surgical correction of the bronchial stenosis is most probably due to an associated but independent vascular malformation. However, anatomical fixation of reflex vasoconstriction cannot be excluded. For everyday practice, the case underlines the importance of excluding a central bronchial stenosis in the "syndrome of unilateral hyperlucent lung" by endoscopy or computed tomography even when clinical and conventional radiological signs of airway obstruction are absent.

\section{References}

1. Di Lorenzo M, Collin PP, Vaillancourt R, Duranceau A. Bronchogenic cysts. J Pediatr Surg 1989; 24: 988-991.

2. Suen HC, Mathisen DJ, Grillo HC, et al. Surgical management and radiological characteristics of bronchogenic cysts. Ann Thorac Surg 1993; 55: 476-481.

3. Bolton JW, Shahian DM. Asymptomatic bronchogenic cysts: what is the best management? Ann Thorac Surg 1992; 53: 1134-1137

4. Kravitz RM. Congenital malformations of the lung. Pediatr Clin North Am 1994; 41: 453-472.

5. Berkovitz KA, Fleischmann JK, Smith RL. Bronchogenic cyst causing a unilateral ventilation-perfusion defect on lung scan. Chest 1988; 93: 1292-1293.

6. Selke AC, Belin RP, Durnin R. Bronchogenic cyst in association with hypoplasia of the left pulmonary artery. J Pediatr Surg 1975; 10: 541-543. 